\title{
POSSIBLE APPLICATIONS OF ADRENOCORTICOTROPHIC HORMONE (ACTH) AND CORTISONE IN SYPHILIS*
}

\author{
BY \\ G. O. HORNE \\ From the General Infirmary, Leeds
}

\section{INTRODUCTION}

Pituitary adrenocorticotrophic hormone (ACTH) and cortisone have already become established as important therapeutic agents in many diseases, and an enormous literature has accumulated since their effect in rheumatoid arthritis was announced by Hench and others (1949). After an initial wave of uncritical enthusiasm, the applications and limitations of these hormones are now being realized, but there are still many gaps in the understanding of their mode of action, both in the normal subject and in disease states.

It is natural that ACTH and cortisone should have been tried in a large number of diseases, and surprising that so far there has been no paper published on their application in human syphilis apart from its ocular manifestations, and only two papers $\dagger$ on their effect in experimental animal syphilis. It may be, of course, that syphilologists in America, who have presumably had access to liberal supplies of the hormones, have been more hesitant than some of their colleagues to use them without substantial justification ; or perhaps their confidence in penicillin (which appears to be greater than ours in Great Britain) leads them to believe that there is no need for improvement in the treatment of syphilis.

However, as has been stressed on many occasions,

* Report of an address delivered to the Medical Society for the Study of Venereal Diseases on January 25, 1952. Received for publication March 11, 1952 .

+ There are reports of four patients with syphilis receiving ACTH incidentally while the effect of the hormone on other conditions was being observed.

Kass, Geiman, and Finland (1951) gave ACTH to three patients Kass, Geiman, and Finland (1951) gave ACTH to three patients
with "evidence of active syphilis of the central nervous system" whith " evidence of active syphilis of the central nervous system" allowed to have three paroxysms and then ACTH was administered during the next two or three. Two of the patients showed "no untoward reaction and no significant changes in behaviour" ; the other, however, became so violent that isolation was required, although he returned to normal 3 weeks after ACTH was stopped. Prior to treatment he had been noted to have mild paranoid tendencies, but had not been agitated or difficult to manage. No other observations from the point of view of syphilis were made.

Clearkin (1952) observed the effect of ACTH in one patient with haemolytic anaemia, who also had a positive blood Wassermann reaction, but apparently no clinical signs of syphilis. He stated that there was no alteration in the blood serology after treatment, but no other observations from the point of view of syphilis were recorded. it is important to regard these newly-isolated hormones not only as therapeutic agents, but as research tools. Few diseases are less understood than syphilis, and there remain unsolved many problems related to its course both in man and in experimental animals. Although penicillin has proved the most effective drug for the treatment of syphilis, its application has failed to solve these problems, and has indeed created some new ones. In considering the possible role of ACTH and cortisone in syphilis, therefore, it is as important to discover whether they can offer any contribution to the understanding of the disease, as it is to determine whether they have any part to play in its therapy.

The more potent a therapeutic agent the more necessary it is to understand its mode of action as well as the disease in which it is being used. Since many of the body tissues and metabolic processes are influenced by ACTH and cortisone, it is obviously necessary to study their action before speculating on what their effect might be on any particular disease. No excuse is therefore required for devoting a considerable proportion of this paper to a summary of what is already known about ACTH and cortisone. Such a summary must be very brief, and will be confined to more or less established facts, with only few references to the literature.

\section{PART ONE}

Adreno-Cortical Hormones.-Three different groups of hormones are secreted by the adrenal cortex; each has certain characteristic effects, although they probably overlap to some extent :

(i) hormones principally concerned with sex (such as adrenosterone) ;

(ii) hormones that influence principally electrolyte balance (such as desoxycorticosterone);

(iii) hormones that influence principally the metabolism of carbohydrate, protein and fat (including cortisone -also called " compound E"--and "compound F").

Those hormones which have been analysed or synthesized have been shown to have a specific 
chemical formula, and because of the nature of their structure are sometimes called "steroids". The chemical name of cortisone is 17-hydroxy-11dehydrocorticosterone.

The secretion of these cortical steroid hormones is controlled by the adrenocorticotrophic hormone of the anterior pituitary (ACTH). When this hormone is secreted, or when a preparation of it is injected into the body, steroids of all three groups are secreted by the adrenals, although the cortisone group predominates. The effect of cortisone can therefore be produced by stimulating the secretion of $\mathrm{ACTH}$, by injecting $\mathrm{ACTH}$, or by injecting cortisone. The first two methods will be successful only if the necessary pathways and the adrenal cortex are intact.

The secretion of ACTH is in turn controlled by the higher centres of the brain, but a large number of factors ultimately influence the secretion of cortisone. Some factors are agents of "stress" ; forms of "stress" which have this effect include physical exertion, disease, and emotion. Another form of "stress" known to stimulate the secretion of ACTH is fever induced by such methods as malaria, intravenous vaccines, and diathermy. During pregnancy also there is an increased secretion of steroid hormones, and it was the remissions of rheumatoid arthritis that frequently occur in pregnancy (and also in jaundice) that helped to stimulate the research that led to the discovery of cortisone.

Principal Physiological and Pharmacological Effects.-ACTH and cortisone influence the metabolism of carbohydrate, protein, and fat. They cause an impairment of tolerance for carbohydrate, which can be demonstrated by blood-sugar curves and insulin sensitivity tests, and which may lead to glycosuria and even to frank diabetes. Studies on nitrogen balance and the urinary excretion of uric acid, creatine, and creatinine indicate that there is an increase in the catabolism of protein while ACTH or cortisone is being administered. The breakdown products are synthesized into liver glycogen, and this is the basis of the most specific method of assay of the hormones, but the technique is unfortunately not clinically applicable. The nature of the disturbed metabolism of fat is less well understood, but its occurrence is shown by the alteration in the bodily distribution of fat, and rarely by ketonuria.

ACTH and cortisone affect the metabolism of water and electrolytes, but apparently in a variable and unpredictable way. There may be a retention of salt and water, sometimes with resultant œdema, or an increased excretion of these substances with a resultant diuresis. There is usually an increased excretion of potassium with the development of a negative potassium balance, and sometimes alkalosis.

$\mathrm{ACTH}$ and cortisone modify the structure and function of the adrenal cortex. The former causes stimulation of the gland with resultant hypertrophy and hyperplasia of the cortical tissue, which returns to normal after withdrawal of the hormone. Cortisone has the opposite effect, since it reduces the necessity for the gland to function, and this depressed function can be demonstrated in several different ways, including the anatomical atrophy of the glands. This may persist for a long period after the withdrawal of cortisone and thus lead to symptoms.

ACTH and cortisone diminish lymphoid tissue and reduce the number of circulating lymphocytes, but more dramatic and more constant is the effect of ACTH in reducing the number of circulating eosinophil granulocytes. This is the basis of one of the tests used for estimating the potency of the hormone preparations and the adequate functioning of the adrenal cortex. The hormones affect the plasma proteins, and especially tend to correct the albuminglobulin ratio when this is disturbed.

$\mathrm{ACTH}$ and cortisone influence the urinary excretion of steroid hormones in a variable, and as yet not completely understood, way. They also have androgenic effects (e.g. masculinization in women) and influence menstrual function (production of amenorrhoea). A large variety of psychic effects have also been observed in patients undergoing treatment with the hormones, ranging from euphoria to true psychotic states.

Cortisone profoundly affects tissues which are the site of inflammation. It reduces the increased capillary permeability characteristic of inflammation, and inhibits the associated exudative phenomena. It inhibits cellular infiltration, formation of granulation tissue, and the vascularization that normally follows inflammation, especially in the cornea. Fibroblastic activity is repressed, and wound healing is delayed, although epithelialization may not be impaired. The delay in wound healing is more marked in some animals than in man, and appears to be variable even in man. Formed fibrous tissue is not affected, and there are conflicting reports on the effects on granulation tissue.

Cortisone interferes with antigen-antibody reactions, such as the Arthus phenomenon (when antigen is injected locally into an animal with circulating antibody, or antibody is injected locally into an animal with circulating antigen, there is loçal tisssue swelling and oedema). Such phenomena 
are directly relevant to the treatment of hypersensitivity states, but the subject of immune reactions is a difficult field of investigation, and, since there are marked differences in the effects of cortisone on various species, experimental reports are conflicting.

The degree to which these physiological and pharmacological effects develop depends partly on the size and duration of the dosage, but there is a wide range of individual variation. Nevertheless it is obvious that, although some may have beneficial therapeutic results, many are undesirable, and some potentially dangerous. Some of the manifestations of hyperadrenalism (Cushing's syndrome) not infrequently develop in patients under treatment with cortisone, and, though these usually disappear after the withdrawal of the hormone, some of the more serious, such as diabetes mellitus, may persist.

Application.-ACTH and cortisone are obviously of value in diseases in which there is a deficiency of these hormones, such as Addison's disease and panhypopituitarism. But they are also beneficial in many diseases in which there is no evidence of such deficiency, which indicates that an excess of the hormones is necessary under certain circumstances, and that their effect is not simply that of "replacement therapy". Indeed, their effects in human disease cannot always be explained by their known physiological and pharmacological actions.

Beneficial results have been observed in two principal types of disease :

(i) the so-called "collagen" diseases (in which the main feature appears to be a disturbance of the structure of collagen), including rheumatoid arthritis, Still's disease, lupus erythematosus, periarteritis nodosa, and scleroderma ;

(ii) " allergic" diseases such as asthma and urticaria, and diseases associated with a hypersensitivity state, such as drug hypersensitivity reactions and a wide range of inflammatory conditions of the eye.

The value of cortisone in the treatment of syphilitic inflammatory ocular disease (interstitial keratitis and iritis) is already established. Since my review of the literature and report of five such cases (Horne, 1951), several other reports have been published (Geddes and McCall, 1950 ; Agatston, 1951 ; Arruga, 1951 ; Crane and McPherson, 1951 ; Duke-Elder and others, 1951 ; Gordon, McLean, Koteen, Bousquet, McCusker, Baras, Wetzig, and Norton, 1951 ; Lavery, Werner, O'Donoghue, Guinan, and Macdougald, 1951 ; McLean, Gordon, and Koteen, 1951 ; Pinkerton, 1951 ; Simpson, Rosenblum, Wood, and Stammer, 1951 ; Werner and Lavery, 1951; Woods, 1951a).
ACTH and cortisone have been used in several other conditions that are within the sphere of venereologists. Cortisone has apparently been successful in four cases of Reiter's syndrome in which it has been used (Brown, Merchant, Wichelhausen, and Robinson, 1951 ; Ogryzlo and Graham, 1950). In two cases of Stevens-Johnson syndrome (the severe form of erythema multiforme exudativum in which genital lesions are sometimes prominent) ACTH was dramatically successful (Bleier and Schwartz, 1951; Wammock, Biederman, and Jordan, 1951), but since similar success has been claimed with aureomycin, no conclusion can yet be drawn. In cases of iritis attributed to gonorrhoea, cortisone has been shown to be as effective as in similar cases of different aetiology (Duke-Elder and others, 1951).

These hormones have also been used in many other diseases, sometimes on the grounds of their known physiological and pharmacological effects, and sometimes empirically. The results vary, and in many such diseases it is still not possible to assess the effects of the hormone.

Mode of Action.-It is not known exactly how ACTH and cortisone produce their effects in disease states. It has already been stated that they do not act as "replacement therapy", except when there is a deficiency of the hormones. That they probably act independently of the development of circulating antibody is shown by the fact that the effects are produced within a few hours of administration when the hormones are given parenterally; cortisone also acts rapidly even when administered locally, as for example, in the eye.

ACTH and cortisone are not bactericidal, and do not "cure" diseases, but simply control the tissue reactions characteristic of certain diseases; they have no direct influence on the noxious agent, but only depress the cellular reaction to it. This effect is illustrated most clearly in ocular inflammatory conditions, when the gross changes may readily be observed to resolve after the local application of cortisone, and the detailed changes may be studied with the slit lamp. It is important to note that, in conditions not themselves self-limiting, withdrawal of the hormone may result in relapse of the condition.

Methods of Administration.-ACTH can be administered by intramuscular or intravenous injection. The former method is usually employed, but intravenous injection is sometimes effective when intramuscular injection is not. Cortisone and cortisone acetate are similar but not identical in 
their action, and discrepancies in reports may be partly due to this. Cortisone is equally effective when administered by intramuscular injection or by mouth. It can also be applied locally under certain circumstances-by drops, ointment, or subconjunctival injection in diseases of the anterior segment of the eye ; by intra-articular injection in rheumatoid arthritis; by ointment in psoriasis and other skin conditions; or by spray in allergic rhinitis. Fairly frequent administration is usually necessary, but "depot" and long-acting preparations of ACTH are now also being employed successfully.

The method of administration, dosage, and duration of treatment vary according to many circumstances ; optimum schedules have yet to be decided on, and indeed must always be adapted for individual cases.

ACTH and Cortisone in Acute Infections.Although a number of conclusions can safely be drawn about the value of ACTH and cortisone in certain "chronic" and allergic diseases, much less is known about their place in the treatment of acute infections. Hyperadrenalism, some degree of which frequently accompanies the administration of these hormones, has long been known to be associated with an increased susceptibility to infection. There is already evidence that tuberculosis may be caused to flare up by ACTH and cortisone, and the progress of some other acute bacterial and virus infections may be considerably modified, sometimes with deleterious results for the patient.

The hormones sometimes interfere with the development of immunity, and organisms are allowed to multiply unrestrained. The deceptive effects of cortisone can be seen best in pulmonary tuberculosis. During treatment with cortisone the patient feels much better, symptoms are reduced, the body temperature returns to normal, and the blood sedimentation rate falls. All these give the impression that the disease is improving, although the sputum is loaded with tubercle bacilli and the disease may be steadily progressing. Sometimes also a " rebound" phenomenon is observed, the disease becoming very much worse after withdrawal of the hormone.

ACTH and cortisone may eventually prove to be of value as an adjunct to treatment of acute infections, especially when a potent antibacterial drug is available and is used coincidentally, but since they are not bactericidal and appear to modify the normal defence mechanisms of the body so that the usual manifestations of disease may not be observed, they are potentially dangerous.

\section{Part Two}

With the foregoing knowledge it is now possible to speculate with reasonable confidence on the possible applications of ACTH and cortisone in syphilis, both from the point of view of therapy and from that of the further elucidation of the natural history of the disease.

In the succeeding discussion it will be necessary to condense the arguments and to make a number of ex cathedra statements about syphilis without supporting them by documented evidence. Some of the latter are so well established as not to require justification in this way, and other conclusions have been made as a result of personal observations and study of the relevant literature. Several of these topics may eventually. justify the publication of more detailed analyses from which the conclusions have been made.

During the discussion the word " cortisone" will be used to include the effect of the hormone when it is mediated by ACTH, except when there is occasion to differentiate between the two.

Early Stages of Syphilis. - What might be the effect of cortisone in the early stage of syphilis, when the disease in many ways resembles other acute infections? Since the "primary" lesion and satellite adenitis represent the local reaction of the tissues to the multiplication of the Treponema pallidum, and the incubation period represents the time necessary for this reaction to develop, both of these may be modified by cortisone. The hormone suppresses tissue reaction of this type; thus the incubation period may be prolonged for as long as cortisone acts, and the chancre may be considerably modified if it develops at all. Since the various " secondary" manifestations of syphilis represent the reaction of the tissues to the widespread multiplication of T. pallidum, these also may be modified.

As has been stated, fever acts as a form of "stress" and results in the secretion of cortisone. Numerous experiments and clinical observations on the effect on syphilis of fever alone (produced by various means, such as hot baths, intravenous vaccines, malaria, and electrical techniques) show that in animals it may prolong the incubation period of syphilis, and even prevent the appearance of syphilomata ; in animals and in man fever therapy may be followed by the partial and even complete healing of " primary" and " secondary" lesions, although in man the latter nearly always recur after the cessation of the fever therapy. When the lesions recur they are sometimes of the so-called " precocious tertiary" type, with severe and extensive destruction of the skin and mucous membranes. 
These effects observed after fever therapy of early syphilis are what might be expected from cortisone. There would be a tendency to suppression of the characteristic lesions, and they might even heal if cortisone were administered after their appearance. Cortisone is not bactericidal, and therefore multiplication of organisms is likely to continue while it is being administered. With certain types of organisms, general symptoms might be produced by the toxins they liberate (although even these symptoms might be suppressed), but since there is little evidence that a toxin is liberated by $T$. pallidum, early syphilis might be rendered completely asymptomatic. The potential dangers of such a situation are obvious. On the other hand, after the withdrawal of cortisone the unprepared tissues might react violently to the presence of numerous circulating spirochaetes, and diffuse and extensive lesions result. A similar situation appears to develop when immunity is impaired, either as a result of the amputation of the primary lesion soon after its appearance, or when a subcurative amount of treatment is given early in the course of the disease.

Support for these speculations is provided by two papers: Turner and Hollander (1950) observed that when cortisone was given to rabbits inoculated with $T$. pallidum the syphilomata showed striking alterations from the picture usually observed in experimental syphilis. There appeared to be a dissociation of parasite and host, in that though the spirochaetes multiplied freely the usual tissue reaction did not occur. The syphilomata became soft and spongy, and there was an excessive accumulation of a mucoid material, tentatively identified as hyaluronic acid. When cortisone was withdrawn there was a " rebound " phenomenon, in which the lesions enlarged to an unusual size. The Wassermann reaction seemed to be inhibited, but in a personal communication Turner has stated that subsequent experiments showed that in many cases even with large doses of cortisone there was no substantial effect either on the Wassermann antibody or on the immobilizing antibody (Turner, 1951).

Heyman, Sheldon, and Evans (1952) injected rabbits with skin syphilomata with ACTH (in daily doses of $5 \mathrm{mg} . / \mathrm{kg}$.) and found a marked decrease in the intensity of the inflammatory and reparative processes.

The polymorphonuclear leucocytes were reduced in number and the collections of lymphocytes at the periphery of the lesions were less extensive. There was no decrease in the number of large mononuclear cells. New formation of fibroblasts and capillaries was diminished and the central oedematous core of the lesions had largely disappeared.
- Since pregnancy is associated with an increased secretion of adrenal cortical hormones, confirmation ought also to be obtained from this source. It is known that the course of syphilis tends to be milder in women than in men, and in particular that pregnancy has an ameliorating effect on the disease. The early signs of syphilis are notoriously suppressed when the disease is contracted in early pregnancy, and animal experiments also show that pregnancy suppresses the clinical manifestations of syphilis. This analogy, however, leaves several problems unsolved. The termination of pregnancy is not usually followed by the development of clinical signs in the mother, and, indeed, even in untreated cases, statistical analyses show that the whole subsequent course of the disease is modified, the incidence of later manifestations being less than might be expected. If the suppressive influence in pregnancy is cortisone, it is difficult to explain this phenomenon by the knowledge available, but of course other hormonal influences are also exerted in pregnancy. It is of interest to note that in one large series of cases an acute febrile illness appeared to have a similar prolonged protective effect. It may also be asked how it is that the mother may show no evidence of syphilis during or after pregnancy (except in serological tests) and yet the foetus may be heavily infected, and also why the foetus is not infected before about the fifth month. Why do the circulating hormones have no protecting influence on the developing foetus, and why in subsequent pregnancies may the same situation occur? The physiology of the adrenal cortex in the new-born infant is not completely understood, but there is evidence that full functional capacity may not be achieved until several weeks after birth. On the other hand, it has been suggested that the action of the cortex is inhibited in the early stages by the circulating maternal steroids. All these problems associated with pregnancy would appear to be more intimately connected with the reaction of the tissues of the mother and foetus than with any particular properties of the spirochaete.

At this point it is of interest to note the observation which has been recorded at frequent intervals in the literature, and has recently been proved statistically, that the combination of fever therapy and specific drugs enhances the cure rate in early syphilis. Various theories have been propounded to explain this phenomenon, but it is obviously worth considering whether it may not result from the stimulation of the adrenal cortex. Might cortisone plus penicillin prove an even more effective remedy for early syphilis than penicillin alone? Cortisone might allow the drug easier access to the organisms, 
especially in view of the nature of the tissue reaction in syphilis. It is conceivable too that, since most organisms are more susceptible to drugs while they are rapidly dividing, cortisone might be of value in this indirect way.*

Before considering the possible effects of ACTH and cortisone in the later stages of syphilis, reference should be made to the so-called "precocious tertiary" lesions which occasionally occur when early syphilis is inadequately treated, and to the relatively rare condition called "malignant" syphilis. In both of these it seems likely that the normal protective reaction of the tissues is much impaired, and so the administration of cortisone might be of value in controlling such lesions until the fundamental infection is eliminated by specific therapy.

Later Stages of Syphilis. - If one subscribes to the view that all the so-called " tertiary" manifestations of syphilis are fundamentally due (at least in part) to hypersensitivity of the tissues to the spirochaete, then cortisone would be expected to have a considerable influence at this stage of the disease. It is obviously not possible to review here the evidence in favour of this conception of the late manifestations of syphilis, but there are good grounds for believing that the lesions of the skin, aorta, central nervous system, and elsewhere, have a common pathological basis. The classical gummatous lesions, especially of the skin, however, differ in their rapid response to specific treatment from the characteristic refractoriness to treatment of general paralysis of the insane, tabes dorsalis, optic atrophy, aortitis, and ocular lesions. It is not possible to assess to what extent this refractoriness is due to the difficulty in penetration of drugs into the areas of tissue reaction, and the matter is complicated by the existence of the blood-brain and blood-aqueous barriers, although aortitis is of course independent of such a possible handicap.

It seems probable that at this stage cortisone would control the tissue reaction, which is characterized by a severity out of proportion to the number of organisms demonstrable. If it did so, then the degenerative changes which follow this inflammatory reaction would be restrained, and

\footnotetext{
* This is supported by the observations of Eagle, Magnuson, and Fleischman (1947), who found that, in vitro, although there was a sevenfold increase in the number of viable organisms after $24 \mathrm{hrs}$ of incubation at $39.7^{\circ} \mathrm{C}$., the organisms died several times faster in the presence of penicillin than after incubation at $37^{\circ} \mathrm{C}$. The spirochaeticidal action of the drug appeared to be enhanced at the higher temperature, despite the favourable effect of the latter on the rate of multiplication of the organisms. There are several possible rate or multiplo this phenomenon, but it may have been due to increased susceptibility of the organisms while they were rapidly increased
dividing.
}

such potentially serious conditions as optic atrophy and aortitis would be arrested: The tissue hypersensitivity would of course be controlled only for so long as cortisone was acting, but it is possible that the coincidental administration of specific drugs would ultimately eliminate the factor responsible for the sensitization. The withdrawal of cortisone might of course be accompanied by important tissue changes, which would be potentially dangerous in such sites as the aorta.

It is possible, too, that cortisone might allow easier penetration of the granulation tissue type of reaction of late syphilis and so enhance the effect of specific drugs. Cortisone would not be expected, of course, to repair damage already done by the degenerative and fibrotic sequelae of the inflammation.

As in early syphilis, such speculations are supported by analogous clinical experience. There are records in the literature of the rapid healing of gummatous lesions of the skin following fever therapy alone, and of healing of similar lesions which had been resistant to specific treatment, when fever therapy was added. The value of fever therapy in those forms of syphilis of the central nervous system which are notoriously refractory to specific treatment has long been established, and it has not been discarded in Great Britain even since the introduction of penicillin.

It is of interest to note that in general paralysis malaria therapy alone has frequently been reported to produce a complete cure of paralysis, as judged by permanent clinical and serological remission. Many theories have been propounded to explain the effect of fever in neurosyphilis, and, since fever acts as an agent of "stress", its effectiveness may be associated with the resultant circulation of cortisone. Fever therapy has rarely been tried in cardiovascular syphilis because of the undesirable physical strain imposed by the methods used, and it is not therefore possible to assess its effect in such lesions.

It has been shown that interstitial keratitis and irido-cyclitis, two of the ocular manifestations of late syphilis in which fever therapy has proved superior to specific therapy, are controlled by cortisone. There is, of course, evidence that these particular manifestations of late syphilis are more characteristic of tissue hypersensitivity than the rest, and it is therefore perhaps hardly justifiable to conclude that cortisone would have the same effect on them all, though there are good grounds for so doing.

The analogy of pregnancy is not available at this stage of the disease, since it rarely occurs after the development of the late manifestations of syphilis. 


\section{Other Possible Effects}

Herxheimer Reaction.-The exact explanation of this phenomenon is not known, but it would seem theoretically possible that cortisone would control its local manifestations. Experimental work by Heyman and others (1952), however, indicated that ACTH did not inhibit the morphological changes of the reaction. The Herxheimer reaction is rarely of clinical importance but any measure known to prevent it would be of definite value, and further study of the effect of cortisone might help to elucidate its nature.

Therapeutic Paradox.-In view of the known effect of cortisone in preventing the formation of fibrous tissue, it might also have a part to play in preventing the therapeutic paradox on the few occasions when this is of clinical importance. It is difficult to speculate on the exact way in which cortisone might be combined with specific therapy in order to retard the too rapid healing which occasionally has undesirable sequelae.

"Sero-Resistant Syphilis".--If this indicates the persistence of spirochaetes in the body, and the reversal of the serological tests is desirable, the combination of cortisone and specific treatment might achieve this end. "Stress", in the form of fever therapy, sometimes helps to reverse these serological tests. Care would be needed in interpreting serological tests in syphilis treated with cortisone lest its effect on immune reactions and on the plasma proteins of the blood should cause confusion. It may be asked whether the wellknown occurrence of fluctuating titres of serological tests in late syphilis might be associated with the circulation of endogenous cortisone?

False Positive Serological Tests.-The possibility that these might be caused by cortisone in patients who do not have syphilis must also be borne in mind. By analogy, it is known that certain febrile illnesses, pregnancy, jaundice, and the development of immune states (as for example, after vaccination), are sometimes associated with false positive tests. These are not necessarily due to cortisone, of course, and may simply be associated with disturbances of the plasma proteins due to other causes.

Reactions to Arsenic, Bismuth, and Penicillin.Here it is fairly safe to assume that cortisone would be of value, especially in the more severe reactions such as exfoliative dermatitis. It would obviously also be of value in such conditions as Addison's disease, and the nephrotic syndrome, when the underlying cause is syphilis.
Cortisone in Ocular Syphilis.-Speculations are not now necessary on the value of cortisone in syphilitic interstitial keratitis and irido-cyclitis, although many problems related to its use remain, such as the best way of administering the hormone and the duration of treatment required in individual cases. As in most diseases treated with ACTH and cortisone, slightly conflicting reports are still coming in, and a complete assessment cannot yet be made. It appears safe to conclude, however, that local application or injection is the method of choice, and that the earlier treatment is started, the better the results. The reiterated warning of Woods that the prohibition of vascularization of the cornea in interstitial keratitis is undesirable (Woods and Chesney, 1946; Woods, 1951b) has yet to be substantiated : recurrence in the same eye and attacks in the other eye occurring after the withdrawal of cortisone are equally well controlled when the hormone is restarted.

It should be realized that other ocular manifestations may also benefit from the hormone. The acute irido-cyclitis that occasionally occurs in "secondary "syphilis usually responds well to specific drug therapy, but cortisone would hasten the relief of symptoms and ensure a better local result. Fever therapy has already been shown to have this effect (Culler and Simpson, 1936; McGavic, 1938; Knight and Schachat, 1946). Choroiditis, chorioretinitis, and neuroretinitis would be expected to benefit from cortisone. Good results have been reported in cases of this type after fever therapy (Culler and Simpson, 1936 ; Knight and Schachat, 1946), and also after ACTH and cortisone, though none of the cases reported in the latter group were associated with syphilis. The possible application of the hormone in optic atrophy has already been referred to.

\section{CONCLUSION}

Some of the possible effects of ACTH and cortisone in syphilis discussed here are highly speculative, but there is little doubt that these hormones influence the natural history of syphilis. It would appear to be desirable to investigate the matter further for three principal reasons :

(i) because the hormones may prove to be valuable in therapy ;

(ii) because of the possible effect on patients with syphilis receiving the hormones for an unrelated disease ;

(iii) because they may help to solve some of the problems related to immunity in syphilis, and so contribute to the study of immunity in general. 
It is important to remember that although ACTH and cortisone are hormones they may be effective when no evidence of hormonal deficiency can be found. The course of syphilis is probably influenced by many complex interacting factors, and it would be unwise to lay too much stress on the influence of hormones. For example, it has frequently been suggested that, since the peak incidence of interstitial keratitis occurs at puberty, the endocrine phenomena of this period are intimately connected with the condition. This may be the case, but the peak incidence of the later manifestations of acquired syphilis occurs at a similar interval after the inoculation of the $T$. pallidum, and the fact that cortisone has proved effective in interstitial keratitis does not necessarily mean that the cause of the disease is basically hormonal.

Speculations have recently been made on the role of hyaluronidase in syphilis, and experimental and human observations suggest that it influences at least the early stage of the disease. Cortisone increases anti-hyaluronidase action in the body, although the significance of this is not yet understood. Whether there is any link between syphilis, cortisone, and hyaluronidase has yet to be shown.

It has often been suggested that the sexual differences in syphilis may be partly attributable to hormonal effects, and the evidence produced in this paper supports this view. This theory might be extended to help to explain the racial differences in syphilis, especially since it is known that there is a relatively. small incidence of the so-called "stress" diseases in the African and oriental races, particularly in less civilized areas. The theory might be extended to help in explaining the whole group of diseases known as "treponematoses". Recent advances in the technique of culturing virulent $T$. pallidum open the way to studying the causative organism of syphilis, but ACTH and cortisone appear to offer opportunities for the equally important study of the reaction of the host to the parasite.

All the possible applications of ACTH and cortisone in syphilis have not been dealt with, and in conclusion a reference is made to a less obvious one. Various observations, which cannot be reviewed here, suggest that trivalent arsenicals may have a cortisone-like effect in addition to their known spirochaeticidal action, and a pilot study at present in progress at Leeds supports this view (Horne, 1952).* If this idea is substantiated, and cortisone is found to be of value in the treatment of

* Here a lantern slide was shown summarizing the data of experiments on five subjects. Using the eosinophil count in the peripheral blood and the urinary uric acid-creatinine ratio as criteria, the intravenous injection of neoarsphenamine produced results similar to those expected from ACTH. syphilis, a useful drug may have been too hastily discarded.

This observation may be of little importance, and in considering the possible applications of ACTH and cortisone in syphilis, enthusiasm must be tempered with discretion. It was recently suggested that in tuberculosis cortisone might be used "to flush the bacilli from lesions while streptomycin and P.A.S. shoot them down ". But it was also pointed out that " to employ such speculative manoeuvres would require great faith in the discrimination of the beaters and the accuracy of the guns " (Brit. med. J., 1951). In attacking syphilis we are blessed with very accurate guns - can we rely on the discrimination of those who direct the beaters?

\section{SUMmaRY}

PART ONE.-The principal physiological and pharmacological effects of ACTH and cortisone are summarized, and their application, mode of action, and methods of administration are discussed.

PART Two.-The possible applications of the hormones in syphilis are discussed. It is pointed out that they may contribute to the understanding of the disease as well as to its therapy. Their possible effects at various stages of syphilis are outlined. The results of experience with cortisone in ocular syphilis are briefly reviewed. The limitations and potential dangers of the hormones are stressed.

\section{AdDENDUM}

Since the delivery of this address, a paper by Sheldon and others (1952) on the effect of ACTH on experimental rabbit syphilis has reached us in Great Britain. It details the observations (including those on the effect on the Herxheimer reaction) referred to briefly by Heyman and others (1952) and quoted above. Some of the conclusions drawn seem to contradict the observations made, but ACTH (in daily doses of $5 \mathrm{mg}$./ $/ \mathrm{kg}$. body weight) undoubtedly diminished the intensity of the inflammatory cell response in the syphilitic lesions and inhibited the proliferative reparative processes.

Robinson (1952) has reported that in three patients with dark-field positive lesions cortisone administered orally resulted in the lesions becoming dry and dark-field negative within 48 hours; withdrawal of cortisone was followed by a rebound phenomenon, the lesions again becoming moist and larger in size. Durel, Sausse, and Borel (1952) have reported that preliminary investigations into the effects of cortisone on the treponemal immobilization test seem to show that the hormone may disturb the test. 


\section{REFERENCES}

Agatston, H. (1951). Amer. J. Ophthal., 34, 1655.

Arruga, H. (1951). Arch. Soc. oftal. hisp.-amer., 11, 585.

Bleier, A. H., and Schwartz, E. (1951). Amer. J. Ophthal., 34, 618.

British Medical Journal (1951). 2, 1570, (annotation).

Brown, T. Mc. P., Merchant, W. R., Wichelhausen, R. H., Robinson, L. B. (1951). "Veterans' Administration Conference on Cortisone Research." A symposium held in August, 1950, at Washington, D.C., p. 52. Merck, Rahway, N.J.

Clearkin, K. P. (1952). Lancet, 1, 183.

Crane, G. W. Jr., and McPherson, S. D. Jr. (1951). Amer. J. Syph., 35, 525.

Culler, A. M., and Simpson, W. M. (1936). Arch. Ophthal., Chicago, 15, 624.

Duke-Elder, S. (1951). Brit. J. Ophthal., 35, 672.

Durel, P., Sausse, A., and Borel, L-J. (1952). British Journal of Venereal Diseases, 28, 68.

Eagle, H., Magnuson, H. J., and Fleischman, R. (1947). Amer. J. Syph., 31, 239.

Geddes, A. K., and McCall, M. F. (1950). Canad. med. Ass. J., 63, 601.

Gordon, D. M., McLean, J. M., Koteen, H., Bousquet. F. P., McCusker, W. D., Baras, I., Wetzig, P., and Norton, E. W. D. (1951). Amer. J. Ophthal., 34, 1675.

Hench, P. S., Kendall, E, C., Slocumb, G. H., and Polley, H. F. (1949). Proc. Mayo Clin., 13, 161.

Heyman, A., Sheldon, W. H., and Evans, L. D. (1952). British Journal of Venereal Diseases, $28,50$.

Horne, G. O. (1951). Brit. med. J., 1, 1289.

-(1952). Unpublished observations.
Kass, E. H., Geiman, Q. M., and Finland, M. (1951). New. Engl. J. Med., 245, 1000.

Kierland, R. R., O'Leary, P. A., Brunsting, L. A., and Didcoct, J. W. (1952). J. Amer. med. Ass., 148, 23.

Knight, H. C., and Schachat, W. S. (1946). Arch. Ophthal., Chicago, 35, 271.

Lavery, F. S., Werner, L. E., O'Donoghue, D., Guinan, P. M., and Macdougald, J. (1951). Brit. med. J., $1,1285$.

McGavic, J. S. (1938). Arch. Ophthal., Chicago, 19, 769.

McLean, J. M., Gordon, D. M., and Koteen, H. (1951). Trans. Amer. Acad. Ophthal. Otolaryng., 55, 565.

Ogryzlo, M. A., and Graham, W. (1950). J. Amer. med. Ass., 144, 1239.

Pinkerton, O. D. (1951). Amer. J. Ophthal., 34, 1746.

Robinson, R. C. V. (1952). British Journal of Venereal Diseases, 28, 80.

Sheldon, W. H., Heyman, A., and Evans, L. D. (1952). Amer. J. Syph., 36, 77.

Simpson, W. G., Rosenblum, B. F., Wood, C. E., and Stammer, E. L. (1951). J. vener. Dis. Inform., 32, 116.

Turner, T. B. (1951). Personal communication.

, and Hollander, D. H. (1950). Bull. Johns Hopk. Hosp., 87, 505.

Wammock, V. S., Biederman, A. A., and Jordan, R. S. (1951). J. Amer. med. Ass., 147, 637.

Werner, L. E., and Lavery, F. S. (1951). Irish J. med. Sci., No. 303, 105.

Woods, A. C. (1951a). Amer. J. Syph., 35, 517. (1951b). Amer. J. Ophthal., 34, 945.

_ _, and Chesney, A. M. (1946). Ibid., 29, 389. 